\title{
Differential expression of microRNAs and their target genes in non-small-cell lung cancer
}

\author{
HUI-YOUNG LEE ${ }^{1}$, SEON-SOOK HAN ${ }^{1}$, HWANSEOK RHEE ${ }^{2}$, JUNG HOON PARK ${ }^{2}$, \\ JAE SEUNG LEE ${ }^{3,4}$, YEON-MOK OH ${ }^{3,4}$, SUN SHIM CHOI ${ }^{5}$, SEUNG-HO SHIN ${ }^{5}$ and WOO JIN KIM ${ }^{1}$ \\ ${ }^{1}$ Department of Internal Medicine, Kangwon National University Hospital, Kangwon National University \\ School of Medicine, Chuncheon-si, Gangwon-do 200-722; ${ }^{2}$ Macrogen Bioinformatics Center, Macrogen, Seoul 153-023; \\ ${ }^{3}$ Department of Pulmonary and Critical Care Medicine and ${ }^{4}$ Clinical Research Center for Chronic Obstructive \\ Airway Diseases, Asan Medical Center, University of Ulsan College of Medicine, Seoul 138-736; \\ ${ }^{5}$ Department of Medical Biotechnology, College of Biomedical Science and Institute of \\ Bioscience and Biotechnology, Kangwon National University, Chuncheon-si, \\ Gangwon-do 200-701, Republic of Korea
}

Received March 21, 2014; Accepted September 9, 2014

DOI: $10.3892 / \mathrm{mmr} .2014 .2890$

\begin{abstract}
MicroRNAs (miRNAs) are single-stranded RNA species that constitute a class of non-coding RNAs, and are emerging as key regulators of gene expression. Since each miRNA is capable of regulating multiple genes, miRNAs are attractive markers for studies of coordinated gene expression. In this study, we investigated miRNA expression profiling using a massively parallel sequencing technique to compare non-small-cell lung cancer (NSCLC) tissue and normal lung tissue. Lung cancer tissue and normal lung tissue were obtained from nine NSCLC patients. RNA isolated from these samples was processed using RNA sequencing (RNA Seq) and the HiSeq 2000 system. Differentially expressed miRNAs and mRNAs were analyzed using a t-test. We selected target pairs that showed a negative correlation among significantly differentially expressed miRNAs and their putative target mRNAs using miRBase Targets. The differences in the expression levels of 222 miRNAs and 1,597 genes were statistically significant, as indicated by an absolute fold change $\geq 1.5$ and $\mathrm{P}<0.05$. miR-577, miR-301b, miR-944, miR-891a and miR-615-3p were generally upregulated, and miR-338-3p was generally downregulated. miRNA-mRNA target pair analysis revealed that 49 miRNAs had 696 target mRNAs. There were significantly differentially expressed miRNAs and mRNAs between lung cancer and normal tissue. Further investigation
\end{abstract}

Correspondence to: Professor Woo Jin Kim, Department of Internal Medicine, Kangwon National University Hospital, Kangwon National University School of Medicine, Baengnyeong-ro 156, Chuncheon-Si, Gangwon-Do 200-722, Republic of Korea

E-mail: pulmo2@kangwon.ac.kr

Key words: microRNAs, carcinoma, non-small-cell lung cancer, genes, sequence analysis of miRNAs and their target genes is warranted to better understand NSCLC.

\section{Introduction}

Lung cancer is the most common cause of cancer-related mortality worldwide. More than 90 million individuals globally are at risk of developing lung cancer, and the disease is suspected to remain a major health problem for many years. The 5-year survival rate is $\sim 10 \%$ (1). More than $75 \%$ of lung cancer cases are diagnosed at late stages, as no practical method of screening the large number of people at risk is available. The late-stage diagnosis is the major contributing factor to the poor prognosis of non-small-cell lung cancer (NSCLC), which accounts for $85 \%$ of all types of lung cancer (2).

To date, cancer genomic studies have focused on the protein-coding genome. However, it is increasingly evident that the non-protein-coding genome also plays a significant role in tumorigenesis (3). The most widely studied class of non-coding RNAs are microRNAs (miRNAs), which are small non-coding RNAs of approximately 22 nucleotides that mediate post-transcriptional gene silencing by controlling the translocation of mRNA into protein. miRNAs are estimated to regulate the translation of more than $60 \%$ of protein-coding genes. Since each miRNA species is capable of regulating multiple genes, miRNAs are attractive markers for studies of coordinated gene expression. In human cancer, the miRNA expression profile differs between normal tissue and tumor tissue, and miRNAs act as oncogenes or tumor suppressor genes and have key functions in tumorigenesis (3). Previous studies have reported differentially regulated miRNAs in diverse solid tumors, including breast (4), lung (5), prostate (6), colon (7) and ovarian (8) cancer.

In solid tumors, miRNAs are deregulated, suggesting their involvement in crucial cellular pathways including cell-cell adhesion and signaling, cell cycle regulation and apoptosis, which play a significant role in the pathogenesis of cancer. 
The majority of these studies were performed using microarrays; however, next-generation sequencing (NGS) of small RNAs provides an efficient platform for the investigation of the ubiquitous and differentially expressed behavior of miRNAs (9). Sequencing has the advantage that all RNAs in a sample, not only those on the chip, may be detected; thus, novel miRNAs that were not reported previously may be identified. Furthermore, we aimed to investigate miRNA regulatory molecules by obtaining information on differentially expressed miRNA-mRNA pairs.

In the present study, we adopted the massively parallel sequencing approach to profile miRNA and provide crucial information with regard to the role of miRNAs in regulating tumorigenesis in NSCLC.

\section{Materials and methods}

Study subjects. The present study used tissue specimens obtained from the Bio-Resource Center of Asan Medical Center (Seoul, Korea) that were donated by nine patients who underwent surgery for NSCLC between March 2008 and March 2011. All of the paired NSCLC and adjacent normal tissue specimens used in the current study were acquired from surgical specimens. Cancer and normal tissue specimens were grossly dissected and preserved in liquid nitrogen immediately after surgery. Appropriate informed consent was obtained from the participants, and the Institutional Review Board of the Asan Medical Center (Seoul, Korea) approved the study (IRB no. AMC IRB 2011-0711).

mRNA expression. Raw data were extracted as fragments per kilobase of exon per million fragments mapped (FPKM) values for each gene from each sample using TopHat and Cufflinks software (10). Data with zeroed FPKM values across all samples and samples with zero values across more than $50 \%$ of the genes were excluded. We added 1 to the FPKM value to facilitate $\log 10$ transformation. Filtered data were logarithm transformed and normalized using the quantile method.

Statistical significance of the expression data was determined using fold changes and Student's t-test or the paired t-test, in which the null hypothesis was that no difference exists between two groups. The false discovery rate (FDR) was controlled by adjusting the P-value using the Benjamini-Hochberg algorithm. Hierarchical clustering was performed using complete linkage and Euclidean distance as a measure of similarity. All data analyses and visualization of differentially expressed genes were conducted using R 2.15.1 (www.r-project.org).

Biological functional annotation analysis of the differentially expressed gene (DEG) list was performed using the DAVID tool (http://david.abcc.ncifcrf.gov/). In the DAVID annotation system, the modified Fisher's exact P-value (EASE score) was adopted to evaluate gene enrichment in annotation terms.

Additionally, a gene set enrichment analysis was conducted for quality control-filtered expression data using GSEA-P (http://www.broadinstitute.org/gsea/index.jsp), which combines information from previously defined sets of genes (MSigDB). Filtered genes were ranked according to the difference in the $\log 10$ expression signal between the case and control using the 'Diff_of_Classes' metric option. The extent of association was measured using a nonparametric,
Table I. Clinical characteristics of NSCLC patients.

\begin{tabular}{lc}
\hline Clinical characteristics & NSCLC patients $(\mathrm{n}=9)$ \\
\hline Age, years (mean \pm SD) & $59(45-68)$ \\
Male, n (\%) & $9(100)$ \\
Smoking (mean \pm SD) & $33 \pm 15.6$ \\
Histological cell type, n (\%) & \\
Squamous cell carcinoma & $5(55.6)$ \\
Adenocarcinoma & $4(44.4)$ \\
TNM stage, n $(\%)$ & \\
I & $4(44.4)$ \\
II & $5(55.6)$ \\
III & $0(0)$ \\
\hline
\end{tabular}

NSCLC, non-small-cell lung cancer.

running-sum statistic termed the enrichment score (ES), and the maximum ES (MES) over all gene sets in the actual data set was rescored from the cases. The significance of the MES score was calculated as the fraction of the 1,000 random permutations for phenotypes. From the analysis, a normalized ES (NES), nominal P-value, FDR q-value adjusting for a gene set size, and correlations between gene sets and actual data were obtained.

miRNA expression. Raw data (the reads for each miRNA) were normalized to the total reads of each individual sample as the standardized to reads per million (RPM; miRNA counts / total count of each sample $\mathrm{x} 1$ million). We excluded miRNAs with zero values across $>90 \%$ of the samples and samples with zero values across $>80 \%$ of the miRNAs. We added 1 to the RPM value to facilitate $\log 10$ transformation. The remaining data processing was as for mRNA analysis.

Integrative (mRNA-miRNA) analysis. We performed mRNA-miRNA data integration for matched samples in each data set.

First, genes and miRNAs whose expression differed significantly (absolute fold change $\geq 1.5$ and $\mathrm{P}<0.05$ ) in each data set were identified. Next, putative mRNA-miRNA target pairs that showed a regulatory association were extracted using mirBase Targets from the Wellcome Trust Sanger Institute (http://microrna.sanger.ac.uk/targets/v5). This approach assumes that the expression of a given miRNA is negatively correlated with the expression of its mRNA targets. We identified negative correlations between any putative miRNA-mRNA pair. For miRNAs with a negative correlation with mRNA, the hypergeometric test was performed to evaluate statistical significance at $\mathrm{P}<0.05$.

Gene ontology $(G O)$ analysis. GO term enrichment analysis for the 696 candidate genes whose regulatory miRNAs were identified was performed using the ClueGo application (ver. 2.0.6) (11) in Cytoscape (ver. 3.0.0) (12). Default parameters were used, and only GO terms with $\mathrm{P}<0.01$ were selected. 


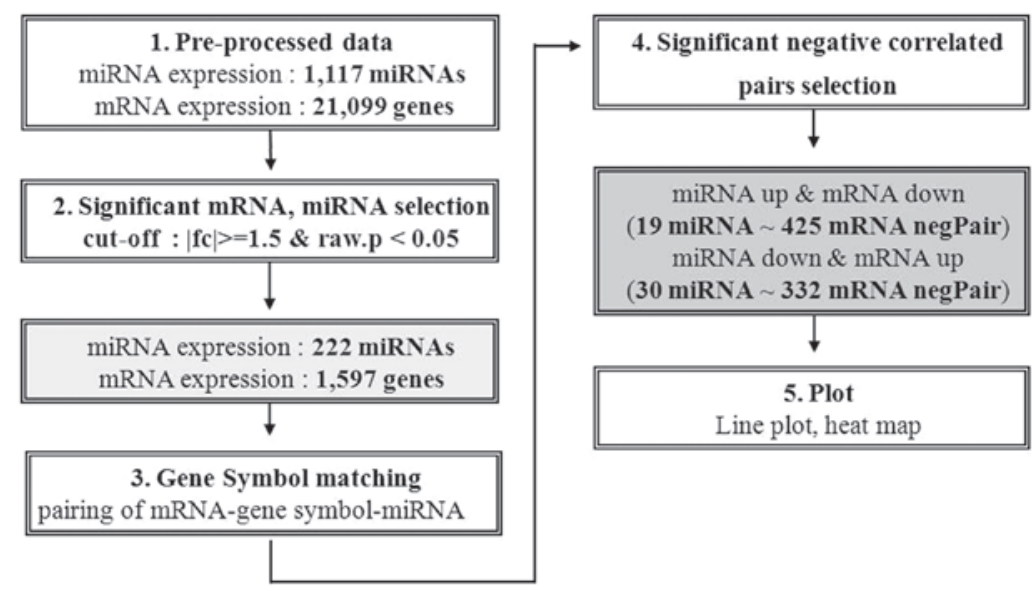

Figure 1. Data analysis flowchart.

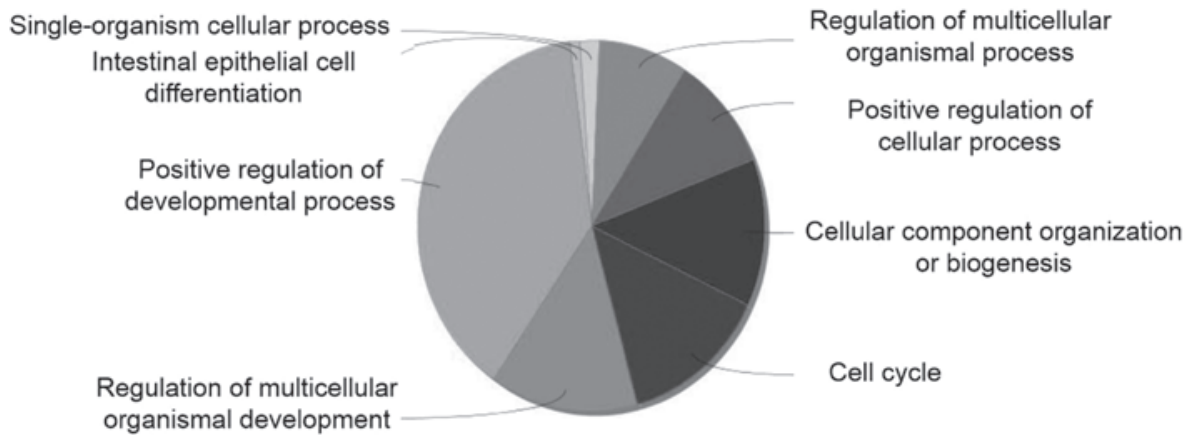

Figure 2. Enrichment analysis for gene ontology terms in the biological process domain. Results of enrichment analysis of 696 differentially expressed genes using the ClueGO application in Cytoscape.

Identification of negatively correlated miRNA-mRNA target pairs associated with the cell cycle. The CluePedia application (ver. 1.0.7) (13) based on the miRBase software (miRanda-m iRNAs-v5-2012-07-19) (14) in Cytoscape was used to investigate the interaction between miRNAs and cell-cycle-related genes identified in the GO enrichment analysis. Among the cell-cycle-related genes, the 100 mRNAs with the highest number of miRNA targets were selected, and negative correlations between the miRNAs and their target mRNAs were investigated.

\section{Results}

Baseline characteristics. Nine patients with completely resected NSCLC were included in our study. All of the patients were male, and the median age was 59 years (range, 45-68 years). The histology consisted of five squamous cell carcinomas (55.6\%) and four adenocarcinomas (42.5\%). The characteristics of the patients are summarized in Table I.

Expressed miRNAs and mRNA profiling. Overall, 1,117 miRNAs and 21,099 genes were differentially expressed in NSCLC and non-cancerous lung tissue. Of these, the differences in the expression levels of 222 miRNAs and 1,597 genes were statistically significant, as indicated by an absolute fold change $\geq 1.5$ and $P<0.05$. miR-205-5p and miR-196a-5p were generally upregulated, and miR-133a and miR-139-5p were generally downregulated. The miRNAs with the greatest degrees of upregulation or downregulation in cancer tissue are listed in Table II.

miRNA-mRNA integrative genomic analysis. We searched for negative correlations between putative miRNA and mRNA pairs; 49 putative negative regulatory miRNA-mRNA pairs were identified. Of these, 19 miRNAs were upregulated and 425 mRNAs were downregulated. Conversely, 30 miRNAs were downregulated and 332 mRNAs were upregulated. Fig. 1 shows the data analysis process. Table III shows miRNAs that have negatively correlated target mRNA. Table IV shows differentially expressed miRNAs in NSCLC and their predicted target genes.

Physical interaction analysis. Fig. 2 shows the enrichment analysis for 696 DEGs using the ClueGO application in Cytoscape. The most relevant GO terms were positive regulation of a developmental process, regulation of multicellular organismal development and cell cycle regulation. Of these, deregulation of the cell cycle underlies the uncontrolled cell proliferation that characterizes the malignant phenotype. We analyzed the interaction between miRNAs and cell-cycle-related genes. Seven miRNAs were identified to have significant negative correlations with their target mRNAs (Fig. 3), as follows: Five miRNAs from the confirmed negative correlations between differentially expressed miRNA-mRNA 
Table II. Top seven up- or downregulated microRNAs in non-small-cell lung cancer.

Fold change

miRNA

(lung cancer/normal tissue)

P-value

\begin{abstract}
Upregulated
hsa-miR-205-5p

hsa-miR-196a-5p

hsa-miR-1246

hsa-miR-577

hsa-miR-301b

hsa-miR-182-5p

hsa-miR-196b-5p

Downregulated

hsa-miR-133a

hsa-miR-139-5p

hsa-miR-144-5p

hsa-miR-338-3p

hsa-miR-1

hsa-miR-490-3p

hsa-miR-30a-3p
\end{abstract}

18.8595

0.0306

11.0968

0.0102

9.2112

0.0111

8.4114

0.0006

7.6426

0.0004

7.4388

0.0015

7.2126

0.0280

$-7.8252$

0.0004

$-7.1165$

0.0002

$-6.5923$

$1.41 \mathrm{E}-06$

$-6.5742$

0.0002

$-6.1193$

0.0002

$-6.1072$

0.0001

$-5.5812$

0.0001

Table III. miRNAs with their putative target genes.

\begin{tabular}{|c|c|c|c|}
\hline miRNA & $\begin{array}{c}\text { miRNA fold change } \\
\text { (lung cancer/normal tissue) }\end{array}$ & miRNA P-value & $\begin{array}{c}\text { P-value } \\
\text { (hypergeometric test) }\end{array}$ \\
\hline hsa-miR-577 & 8.411 & 0.0006 & 0.0461 \\
\hline hsa-miR-301b & 7.643 & 0.0004 & 0.0360 \\
\hline hsa-miR-944 & 5.765 & 0.0422 & 0.0416 \\
\hline hsa-miR-891a & 5.683 & 0.0229 & 0.0020 \\
\hline hsa-miR-615-3p & 5.211 & 0.0064 & 0.0299 \\
\hline hsa-miR-671-5p & 2.606 & 0.0119 & $3.83 \mathrm{E}-06$ \\
\hline hsa-miR-429 & 2.599 & 0.0429 & 0.0007 \\
\hline hsa-miR-210 & 2.246 & 0.0334 & $9.27 \mathrm{E}-06$ \\
\hline hsa-miR-137 & 2.002 & 0.0412 & 0.0143 \\
\hline hsa-let-7c & -2.044 & 0.0105 & 0.0046 \\
\hline hsa-miR-338-3p & -6.574 & 0.0002 & 0.0442 \\
\hline
\end{tabular}

targets (hsa-miR-490-5p, hsa-miR-423-5p, hsa-miR-328, hsa-miR-1 and hsa-let-7); hsa-miR-153, which is expected to regulate forkhead box $\mathrm{M} 1$, a gene identified as a putative hub in the protein-protein interaction network; and hsa-miR-215, which interacts with cell-cycle-related genes in miRcode (15).

\section{Discussion}

In the present study, we identified 222 significantly differentially expressed miRNAs using the massively parallel sequencing approach in human lung cancer tissue compared with normal lung tissue. Furthermore, we identified putative negative regulatory miRNA-mRNA target pairs. Nineteen miRNAs were upregulated, and 425 matched mRNAs were downregulated. Additionally, 30 miRNAs were downregulated, and 332 matched mRNAs were upregulated. miR-577, miR-301b, miR-944, miR-891a, miR-615-3p and miR-338-3p were highly differentially expressed in the current study.

We searched for putative target genes associated with lung cancer. miR-577 was generally upregulated (fold change $=8.411$ ) and negatively correlated with epidermal growth factor-containing fibulin-like extracellular matrix protein 1 (EFEMP1, also known as fibulin-3). EFEMP1 belongs to the fibulin family of widely expressed extracellular matrix proteins that regulate cell proliferation. This family mediates cell-to-cell and cell-to-matrix communication, as well as providing organization and stabilization to extracellular matrix structures during organogenesis and vasculogenesis. Several studies have demonstrated that fibulin-3 and another fibulin family member, fibulin-5, antagonize tumor angiogenesis 
Table IV. Differentially expressed miRNAs in non-small-cell lung cancer and their predicted target genes.

\begin{tabular}{|c|c|c|c|}
\hline miRNA & $\begin{array}{c}\text { miRNA } \\
\text { fold change }\end{array}$ & Target gene & $\begin{array}{l}\text { Gene } \\
\text { fold change }\end{array}$ \\
\hline \multirow[t]{5}{*}{ miR-577 } & 8.411 & GNG11 & -5.143 \\
\hline & & EFEMP1 & -4.578 \\
\hline & & SCEL & -3.625 \\
\hline & & GKN2 & -3.47 \\
\hline & & ATG16L2 & -2.723 \\
\hline \multirow[t]{5}{*}{$\operatorname{miR}-301 b$} & 7.643 & HOXA5 & -4.096 \\
\hline & & SPARCL1 & -4.028 \\
\hline & & HYAL1 & -3.869 \\
\hline & & SERPING1 & -3.825 \\
\hline & & ALOX5 & -3.33 \\
\hline \multirow[t]{5}{*}{ miR-944 } & 5.765 & SYT15 & -3.305 \\
\hline & & SVEP1 & -2.314 \\
\hline & & OMD & -2.645 \\
\hline & & GATA6 & -2.089 \\
\hline & & CYB5A & -2.552 \\
\hline \multirow[t]{5}{*}{ miR-891a } & 5.683 & IL1R1 & -5.836 \\
\hline & & CLIC5 & -5.621 \\
\hline & & SLC27A3 & -4.292 \\
\hline & & HOXA5 & -4.096 \\
\hline & & ITIH5 & -3.498 \\
\hline \multirow[t]{5}{*}{ miR-615-3p } & 5.211 & SFTPC & -43.343 \\
\hline & & PTGDS & -7.008 \\
\hline & & C10orf116 & -6.789 \\
\hline & & ABI3BP & -6.529 \\
\hline & & TAGLN & -4.285 \\
\hline \multirow[t]{5}{*}{ miR-338-3p } & -6.574 & HIST2H4B & 2.862 \\
\hline & & CEACAM1 & 2.705 \\
\hline & & SF3B4 & 2.652 \\
\hline & & ZNF238 & 2.477 \\
\hline & & MCM4 & 2.399 \\
\hline
\end{tabular}

in vivo (16), suggesting that concerted deregulation of a set of antiangiogenic factors, including fibulin-3 and tissue inhibitor of metalloproteinase 3, contributes to tumor progression (17).

miR-301b is negatively correlated with homeobox A5 (HOXA5), which is known to be a tumor suppressor gene whose gene product positively regulates the expression of the TP53 tumor suppressor gene (18). Liu et al suggested that microRNA-196a promotes NSCLC cell proliferation and invasion by targeting HOXA5 (19). However, in our study, HOXA5 was negatively regulated by both miR-301b and miR-891a. Additionally, miR-301b was shown to be negatively correlated with secreted protein acidic and rich in cysteine-like 1 (SPARCL1) and hyaluronoglucosaminidase 1 (HYAL1). SPARCL1, also known as hevin, belongs to the matricellular protein family. SPARCL1 is downregulated in NSCLC (20). Additionally, Yu et al (21) reported that SPARCL1 is likely to be a significant negative regulator in the progression or metastasis of colorectal cancer. HYAL1, which encodes a lysosomal hyaluronidase, was downregulated in NSCLC tissue. Anedchenko et al (22) reported that HYAL1 and HYAL2 genes are downregulated in NSCLC, a finding that is consistent with our data.

miR-944 was negatively correlated with GATA binding protein 6 (GATA6), which is a member of a small family of zinc finger transcription factors that play a significant role in the regulation of cellular differentiation and organogenesis. This protein is expressed during early embryogenesis and localizes to endo- and mesodermally derived cells during later embryogenesis, thereby playing a significant role in lung development. Cheung et al (23) reported that lung adenocarcinoma progression is regulated in part by the lineage transcription factor GATA6.

miR 338-3p was generally downregulated (fold change $=-6.574$ ), and was negatively correlated with the putative target genes carcinoembryonic antigen-related cell adhesion molecule 1 (CEACAM1) and minichromosome maintenance protein 4 (MCM4). CEACAM1, a single-pass transmembrane type I glycoprotein, belongs to the carcinoembryonic antigen 


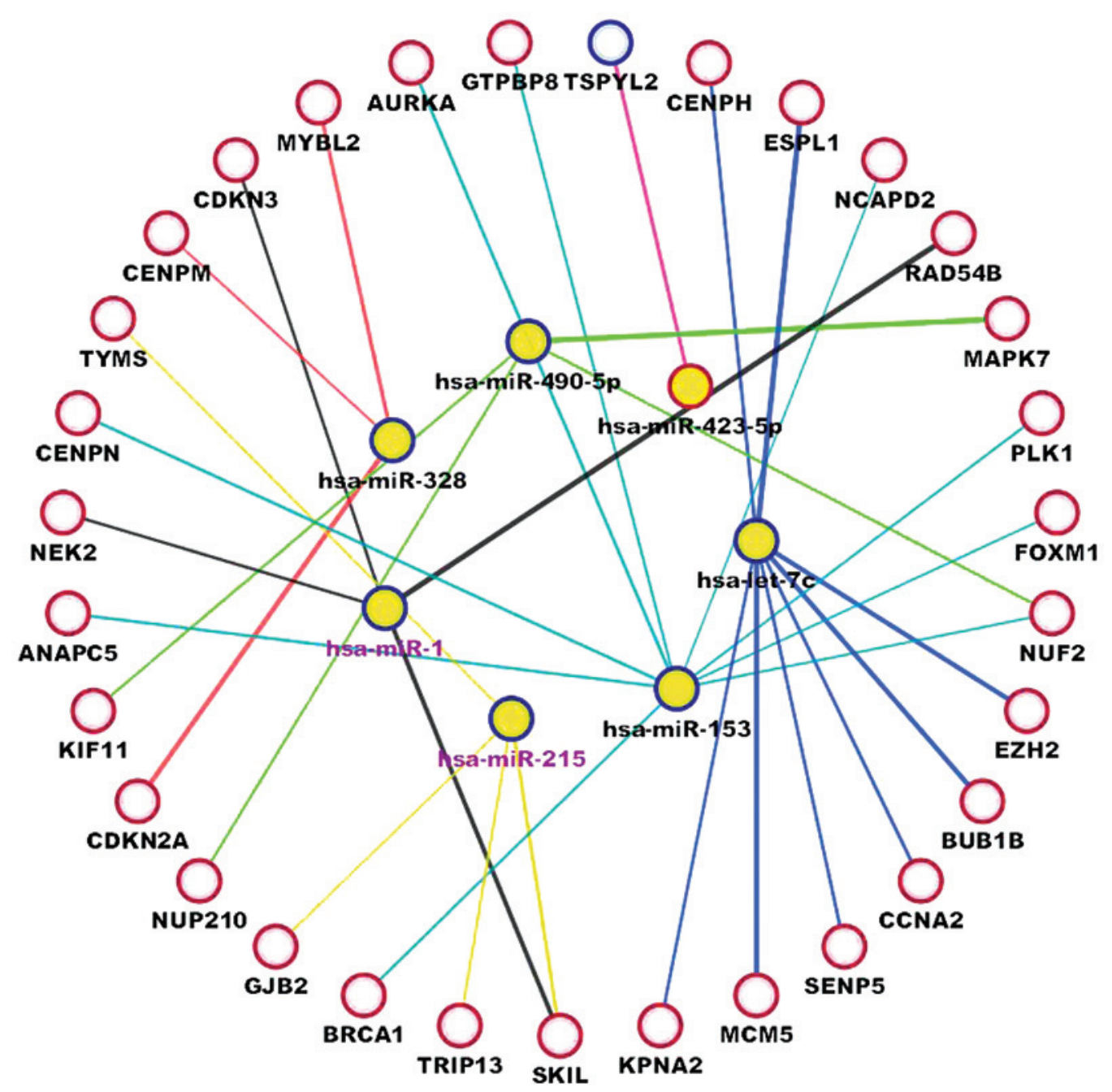

Figure 3. Confirmed negative interactions between miRNAs and their target mRNAs associated with the cell cycle. Using miRNA-mRNA target pair information, seven miRNAs were identified to have the expected negative correlations with cell-cycle-related genes. Red and blue lines in each node (or circle) indicate up- and downregulated genes, respectively. Yellow and white colors inside nodes represent miRNAs and their target mRNAs, respectively. Edge width indicates the $\kappa$ value $(\kappa>0.6)$ as an indicator of the degree of reliability of the edge between two nodes.

family. In lung cancer, accumulated immunohistochemical evidence indicates that epithelial CEACAM1 expression is associated with tumor metastasis and progression (24). Zhou et al demonstrated that CEACAM1 mRNA levels in tumors were higher those in adjacent tumor-free tissues, although not significantly (24). Additionally, MCM4 is one of six MCM proteins comprising the prereplicative complex that binds to replication origins in the G1 phase of the cell cycle and is essential for the initiation of DNA replication. Kikuchi et al (25) reported that MCM4 expression was higher in lung cancer cells than in adjacent normal bronchial epithelial cells $(\mathrm{P}<0.001)$, and high MCM4 expression was correlated with poorer differentiation $(\mathrm{P}<0.001)$.

miR-210 is one of the most consistently reported upregulated miRNAs in human lung cancer miRNA expression profiling studies $(1,26)$. In our study, miR-210 was significantly differentially expressed (fold change $=2.246 ; \mathrm{P}=0.03$ ). miR-210 has a number of validated targets associated with the regulation of mitochondrial metabolism, angiogenesis, cell cycle regulation and X-chromosome inactivation (1), and miR-210 is upregulated by hypoxia inducible factor $1 \alpha$ (HIF-1 $\alpha)$ in response to hypoxic conditions. Recently, miR-210 was reported to increase the radioresistance of hypoxic cancer cells (27).

Our results were partly consistent with those of previous studies of miRNA in lung cancer, and novel miRNAs that may play an active role in cancer development were identified. Our study used the NGS approach to profile miRNA expression in human lung cancer tissue compared with matched normal lung tissue, although only a small number of samples were used. As the technology develops, the cost of sequencing is expected to decline, making it possible for NGS to be widely used in the future. The major advantages of NGS are its high-throughput capability, and that it is precise, accurate and repeatable. Its application includes new miRNA exploration, detection of miRNA, miRNA editing and isomiR and target mRNA detection. NGS of small RNAs facilitates the investigation of the ubiquitous and differentially expressed behavior of miRNAs, and will therefore promote miRNA research.

The current study had several limitations. Few samples were included, and no validation study was performed. Additionally, our study compared tumor and non-cancerous tissue only. The identification of markers associated with 
patient survival, disease prognosis or response to a specific anticancer drug is warranted. However, our results include overlapping miRNAs that were reported previously.

In conclusion, significantly differentially expressed miRNAs and mRNAs between lung cancer and normal tissue were identified by means of massively parallel sequencing. These miRNAs and their target genes may play a significant role in lung cancer. Further studies are warranted to fully understand the pathogenesis of NSCLC.

\section{Acknowledgements}

This study was supported by a grant from the National R\&D Program for Cancer Control (1020420) and the Ministry for Health and Welfare and Basic Research Program through the National Research Foundation of Korea (C1010458-01-01) funded by the Ministry of Education, Republic of Korea.

\section{References}

1. Võsa U, Vooder T, Kolde R, et al: Meta-analysis of microRNA expression in lung cancer. Int J Cancer 132: 2884-2893, 2013.

2. Jemal A, Bray F, Center MM, et al: Global cancer statistics. CA Cancer J Clin 61: 69-90, 2011

3. Esteller M: Non-coding RNAs in human disease. Nat Rev Genet 12: 861-874, 2011.

4. Iorio MV, Ferracin M, Liu GC, et al: MicroRNA gene expression deregulation in human breast cancer. Cancer Res 65: 7065-7070, 2005.

5. Yanaihara N, Caplen N, Bowman E, et al: Unique microRNA molecular profiles in lung cancer diagnosis and prognosis Cancer Cell 9: 189-198, 2006.

6. Porkka KP, Pfeiffer MJ, Waltering KK, et al: MicroRNA expression profiling in prostate cancer. Cancer Res 67: 6130-6135, 2007.

7. Akao Y, Nakagawa Y and Naoe T: MicroRNA-143 and -145 in colon cancer. DNA Cell Biol 26: 311-320, 2007.

8. Yang H, Kong W, He L, et al: MicroRNA expression profiling in human ovarian cancer: miR-214 induces cell survival and cisplatin resistance by targeting PTEN. Cancer Res 68: 425-433, 2008.

9. Sablok G, Milev I, Minkov G, et al: isomiRex: web-based identification of microRNAs, isomiR variations and differential expression using next-generation sequencing datasets. FEBS Lett 587: 2629-2634, 2013.

10. Trapnell C, Roberts A, Goff L, et al: Differential gene and transcript expression analysis of RNA-seq experiments with TopHat and Cufflinks. Nature protocols 7: 562-578, 2012.
11. Bindea G, Mlecnik B, Hackl H, et al: ClueGO: a Cytoscape plug-in to decipher functionally grouped gene ontology and pathway annotation networks. Bioinformatics 25: 1091-1093, 2009.

12. Shannon P, Markiel A, Ozier O, et al: Cytoscape: a software environment for integrated models of biomolecular interaction networks. Genome Re 13: 2498-2504, 2003.

13. Bindea G, Galon J and Mlecnik B: CluePedia Cytoscape plugin: Pathway insights using integrated experimental and in silico data. Bioinformatics 29: 661-663, 2013.

14. Kozomara A and Griffiths-Jones S: miRBase: integrating microRNA annotation and deep-sequencing data: Nucleic Acids Res 39: (Database issue) D152-D157, 2011.

15. Xiao F, Zuo Z, Cai G, et al: miRecords: an integrated resource for microRNA-target interactions: Nucleic Acids Res 37: (Database issue) D105-D110, 2009.

16. Albig AR, Neil JR and Schiemann WP: Fibulins 3 and 5 antagonize tumor angiogenesis in vivo. Cancer Res 66: 2621-2629, 2006.

17. Yue W, Dacic S, Sun Q, et al: Frequent inactivation of RAMP2, EFEMP1 and Dutt1 in lung cancer by promoter hypermethylation. Clin Cancer Res 13: 4336-4344, 2007.

18. Raman V, Martensen SA, Reisman D, et al: Compromised HOXA5 function can limit p53 expression in human breast tumours. Nature 405: 974-978, 2000.

19. Liu X, Lu K, Wang K, et al: MicroRNA-196a promotes non-small cell lung cancer cell proliferation and invasion through targeting HOXA5. BMC Cancer 12: 348-348, 2012.

20. Schraml P, Shipman R, Colombi M and Ludwig CU: Identification of genes differentially expressed in normal lung and non-small cell lung carcinoma tissue. Cancer Res 54: 5236-5240, 1994.

21. Yu S, Yu J, Ge W, et al: SPARCL1, Shp2, MSH2, E-cadherin, p53, ADCY-2 and MAPK are prognosis-related in colorectal cancer. World J Gastroenterol 17: 2028-2036, 2011.

22. Anedchenko EA, Dmitriev AA, Krasnov GS, et al: Down-regulation of RBSP3/CTDSPL, NPRL2/G21, RASSF1A, ITGA9, HYAL1 and HYAL2 genes in non-small cell lung cancer. Mol Biol 42: 965-976, 2008 (in Russian).

23. Cheung W, Zhao M, Liu Z, et al: Control of alveolar differentiation by the lineage transcription factors GATA6 and HOPX inhibits lung adenocarcinoma metastasis. Cancer Cell 23: 725-738, 2013.

24. Zhou M, Du Y, Liu Y, et al: Clinical and experimental studies regarding the expression and diagnostic value of carcinoembryonic antigen-related cell adhesion molecule 1 in non-small-cell-lung cancer. BMC Cancer 13: 359, 2013.

25. Kikuchi J, Kinoshita I, Shimizu Y et al: Minichromosome maintenance (MCM) protein 4 as a marker for proliferation and its clinical and clinicopathological significance in non-small cell lung cancer. Lung Cancer 72: 229-237, 2011.

26. Guan P, Yin Z, Li X, et al: Meta-analysis of human lung cancer microRNA expression profiling studies comparing cancer tissues with normal tissues. J Exp Clin Cancer Res 31: 54, 2012.

27. Grosso S, Doyen J, Parks SK, et al: MiR-210 promotes a hypoxic phenotype and increases radioresistance in human lung cancer cell lines. Cell Death Dis 4: e544, 2013. 